\title{
Cuerpos Múltiples en la Interactividad. Representaciones y trayectos de lo corpóreo.
}

Multiple Bodies in Interactivity. Representations and pathways of the corporeal.

\section{> Andrea Sosa}

Universidad Nacional de La Plata, Argentina

Instituto Universitario Nacional del Arte, Argentina

correo.andreasosa@gmail.com

\begin{abstract}
The present work looks into the place the body occupies within interactive experiences. Firstly, we explore the nature of the machine in its corporeal dimension, and the human body in the digital representation process. Then, a classification is proposed for the modes of articulation of the participant's body in interactivity, following various analytic frameworks. Finally, we analyze the difference between the physical bodies within an experience and the construction of the bodies present in the artwork, outlining the dialogue between technical engineering and the symbolic dimension.
\end{abstract}

Reywords: Body; Interactivity; Interfaces; Representations; New Media.

\section{Introducción}

An interactive work challenges one to undergo a transformation from an onlooker to an "interactor", an active agent. A pecular kind of dialogue develops. In addition to mental interaction that is a precondition to the reception of art in general, pshysical, bodily action -one that involves more than just movement of the eyes-takes place.

Erkki Huhtamo

El Arte Interactivo sacude la tradición espectatorial, invita a los cuerpos a levantarse de un lugar de exclusiva recepción, y asumir un rol activo en el territorio de la obra.

Si observamos detenidamente una obra interactiva, veremos que hay cuerpos en movimiento, que exploran, dialogan, se vinculan sensorialmente con la obra. El ojo directo los ve como cuerpos enteros. Sin embargo, existe una diferencia -frecuentemente inadvertida- entre el cuerpo del interactor que está en el espacio, y el cuerpo integrado en la experiencia interactiva, a través del cual, el público interactúa. Los cuerpos físicos en el espacio difieren de los cuerpos percibidos por el sistema interactivo y los cuerpos interactuantes, y aún así, todos se pliegan y convergen en el sujeto que dialoga con la obra.

Como sostiene Caroline Jones (2005), las mediaciones en la sensorialidad humana se han intensificado fuertemente.

Al cuerpo físico del público inmerso en la experiencia artística se suma el cuerpo maquínico de la obra. Y en el encuentro entre ambos: se cierne un universo semántico que reconfigura la relación entre objetos, sujetos y entornos.

\section{El Cuerpo Máquinico y Sus Sentidos}

Estamos transfiriendo hoy lo que nosotros sabemos sobre las máquinas a los organismos vivos y viceversa. Es por esa razón que a veces nos referimos a los cuerpos como máquinas y a las máquinas, o a los procesos técnicos en general, como a una especie de vida (vida artificial).

Arlindo Machado

Así como el interactor es una unidad mente-cuerpo, el dispositivo informático puede ser entendido como una configuración artificial mente-cuerpo. El binomio hardware-software que da forma a los chips en acción, sugiere la idea de una dimensión física (hardware) y una dimensión intangible, semejante a los procesos mentales (software). En relación al conjunto en su totalidad, podría pensarse al hardware como el cuerpo del sistema interactivo.

En este contexto, los sensores serían a la máquina, lo que los sentidos son al cuerpo humano: puentes entre el interior y el exterior, acceso al intercambio de información, vértices donde se articulan la receptividad de lo externo y la exteriorización de los procesos internos. El nexo lexical entre la palabra sensor y la palabra sentido señala las analogías subyacentes en la constitución de los dispositivos maquínicos. En este sentido, los sensores, como 
ventanas al mundo, le permiten al sistema percibir y entablar una relación con el entorno.

También es posible pensar al cuerpo humano como una interface orgánica. David Rokeby traza una comparativa sobre la diferente naturaleza de nuestro cuerpo y las interfaces:

"Nuestra interfaz "orgánica” es extraordinariamente compleja y masivamente paralela. Nuestro sistema sensorio involucra un número enorme de sensores simultáneamente activos, y actuamos sobre el mundo a través de un número aún mayor de puntos individuales de contacto físico. En contraste, nuestras interfaces artificiales son notoriamente estrechas y seriales ...”. (Rokeby: 1990)

Dada la diversidad de los sensores, son múltiples los sentidos que pueden imbuirse en el hardware. El cuerpo de la máquina es, por definición, un cuerpo polimorfo. Sus sentidos y umbrales estarán definidos por los algoritmos y condicionados por las velocidades de procesamiento. Las características que asuma en cada obra, determinará, a su vez, qué dimensiones de la realidad podrá percibir y con qué cuerpos del público podrá entablar diálogos.

\section{El Cuerpo Orgánico y sus Representaciones}

Alejados de la lógica mecánica e insertos en el nuevo régimen digital, los cuerpos contemporáneos se presentan como sistemas de procesamiento de datos, códigos, perfiles cifrados, bancos de información. Lanzados a las nuevas cadencias de la tecnociencia, el cuerpo humano parece haber perdido su definición clásica y su solidez analógica; en la estera digital se vuelve permeable, proyectable, programable.

Paula Sibilia

El cuerpo físico del público en contacto con la obra interactiva se constituye en soporte de un otro cuerpo: el cuerpo representado, virtualizado, el cuerpo percibido por la obra; ambos cuerpos pueden coincidir o diferir sustancialmente. A continuación, se presentan -con una impronta taxonómica- diferentes modos en que el cuerpo se integra a las obras interactivas.

\section{El cuerpo total}

En algunas obras, la interacción descansa en la acción de un cuerpo unificado. Usualmente se trata de obras que emplean el movimiento en el espacio como variable para la interactividad. Una obra como «Rain Room» (2012), de Random International, invita al usuario a experimentar una curiosa situación: caminar bajo la lluvia sin mojarse. De este modo, la lluvia copiosa que cae en la sala tendrá hiatos espaciales en el punto exacto donde el cuerpo se localice. Los cuerpos transitan y recorren el espacio, habilitando a su paso, un claro de agua que los impermeabiliza, alejando la humedad del contacto con la piel. Aquí, el cuerpo funciona como una totalidad en la experiencia.

El cuerpo es concebido como una totalidad que estimula la obra y desata el devenir de la forma.

\section{El cuerpo parcial}

En diversas obras interactivas, el cuerpo deja de ser una totalidad compacta para ser reinterpretado y parcialmente incorporado a la obra. Así como en la producción cinematográfica, el cuerpo entero de un actor en la puesta en escena es analizado y cercenado visualmente cuando es capturado en un primer plano, en muchas obras interactivas el cuerpo es fragmentado, permaneciendo lo físico como soporte material de la interacción y recortando ciertos sentidos, miembros u órganos para formar parte del cuerpo interactuante en la obra. Establecemos tres ejes de análisis diferentes para los cuerpos parciales.

\section{1- Sentidos}

Si la misión del arte del siglo XX fue hacer visible lo invisible, en el siglo XXI los artistas estarian preocupados en encontrar nuevas formas de permitirnos sentir lo invisible en lo visible. La proporción de los sentidos puede cambiar para que nuevos tipos perceptuales puedan desocultarse

Roy Ascott

Diversas obras construyen su mecánica interactiva a partir de algún sentido en particular.

A) Vista - Ojos

Una obra emblemática de esta aproximación la constituye «Zerseher» (1991) de Joachim Sauter. En el marco de una galería de arte, el otrora espectador, se ubica frente a un cuadro pero algo fuera de lo usual acontece: su mirada irá deconstruyendo la imagen. La obra aborda de lleno la problemática clásica del espectar como fenómeno pasivo y la interactividad devenida de las estéticas interactivas.

En esta obra, el gran punto de contacto de la actividad interactiva lo constituye el ojo, y más precisamente, el movimiento ocular.

\section{B) Oido - Oreja}

Por su naturaleza receptiva, el oído no suele ser un sentido que produzca estímulos de entrada en las obras, pero sí puede observarse que, en algunos trabajos, aparece como un sentido jerarquizado en términos de la conexión entre el cuerpo y la obra. Un proyecto de esta variante es «Hot and Cold Whisperen» (2009) de Ebru Kurbak y Jona Hoier, el cual emula el juego de niños "frío/caliente". La interface consiste en unas orejeras que el usuario se coloca en su cabeza. En el ambiente de la ciudad, hay señales wifi, invisibles e intangibles. Las orejeras obran como detectores de las señales. Una voz susurrará al oído "caliente", "tibio", "frío", en relación a la proximidad o lejanía de una señal wifi, mientras el usuario deambula por el espacio.

\section{C) Gusto - Lengua / Olfato - Nariz}

El gusto es quizás -junto al olfato- uno de los sentidos menos explorados en las obras interactivas. Aunque pocos, existen algunos ejemplos. Podemos citar un trabajo denominado «Mate Parlante» (2013) desarrollado en la Carrera de Artes Multimediales del IUNA (Argentina) por Mariano Ferle, Azucena Lozana y Sebastián Caiafa. El trabajo propone al usuario sentarse a tomar mate. Así, un termo que contiene el agua, permite verter el líquido en el mate que contiene yerba y que oficia de interface. Cuando el usuario vierte el 
líquido, puede, a través de la bombilla, succionar el líquido. En ese gesto, beberá el líquido y a su vez el mate le contará una anédcota sobre la tradición de esta infusión, explorando un rito fundante de la idiosincrasia rioplatense. A través de la propia acción física de beber, accederá a los contenidos de la obra.

D) Tacto - Piel

La inclusión del sentido del tacto es, probablemente, la mayor innovación que ha introducido el Arte Interactivo, en términos de la dimensión sensorial en la obra. Como sostiene Erkki Huhtamo (2007), el Arte Interactivo está ligado a la acción de tocar y esto produce resignificaciones profundas en el campo de la experiencia artística.

La obra «Delicate Boundaries» (2007) de Chris Sugrue, se articula en función del tacto, en más de un sentido. Organismos virtuales contenidos en una pantalla se trasladan al espacio físico, sobre la mano del usuario y podrán recorrer la superficie de los miembros superiores; la piel es el punto de encuentro entre el cuerpo y la imagen.

La obra «Touch Me» (2004) del grupo holandés Blendid integrado por David Kousemaker y Tim Olden, necesitará de la presión del cuerpo sobre una superficie de vidrio esmerilada para dejar una impresión de sí mismo. Casi emulando a un escáner a gran escala, los participantes se ubican contra el vidrio de diversos formas cuando una luz barre la superficie e imprime su cuerpo; en un resultado visual que recuerda las inscripciones realizadas en las cavernas, 10.000 ańos atrás. Gran parte de las obras interactivas contemporáneas incluyen el tacto, en alguna de sus formas.

\section{Miembros}

Otro criterio posible es pensar el cuerpo en términos de sus partes o sus miembros. Este criterio se solapa con los sentidos, pero en principio, el propósito de este eje nace al observar algunas configuraciones que no estarían primordialmente vinculados a los sentidos, aunque los incluyan.

\section{A) Manos}

La obra de Christa Sommerer y Laurent Mignonneau «Mobile Feelings» (2002), pone el énfasis en la mano, como vértice para la interacción. A partir de una interface tangible, un objeto en forma de calabaza, dos usuarios pueden sentir parámetros vitales (ritmo cardíaco y respiración) de la otra persona. De este modo, la obra genera una comunicación no verbal, corporal e intuitiva, entre personas remotas.

Podemos ir incluso más en detalle, y observar algunos casos donde sólo una parte de una mano, puede convertirse en el cuerpo actuante en la obra. En el trabajo «Pulse Index» (2010) de Rafael Lozano Hemmer, un dedo es el punto de contacto entre el público y la obra. El usuario es invitado a ingresar su dedo en una cavidad en la pared. La interface captura una imagen óptica de sus huellas digitales que proyecta, amplificadas, sobre pantallas que circundan al espacio de exhibición. Al mismo tiempo, la interface sensa su ritmo cardíaco e imprime las imágenes a una cadencia análoga al pulso del usuario.

\section{B) Cabeza}

El artista autraliano George Khut desarrolló «Wee Leaf» (2009) en el marco de la residencia "Thinking through the body: Sensorium Gymnasium», donde diversos artistas exploraron la potencialidad del movimiento, el tacto y la propiocepción. En este trabajo una hoja de árbol pende desde el techo, a la altura de la cabeza de una persona. La obra propone al usuario pararse sobre una plataforma capaz de sensar el balance corporal, e induce al usuario a contactar su rostro con la hoja y producir movimientos. Las oscilaciones de la hoja en el espacio, y el balanceo, son traducidas a sonidos, en una situación que propone al cuerpo una experiencia poco usual en las obras interactivas: se interactúa con el rostro prioritariamente, la acción interactiva se localiza en la cabeza como una totalidad.

\section{C) Aparato fonador}

«Universal Whistling Machine» (2004) de Marc Böhlen y JT Rinker aborda el tema de la universalidad del lenguaje, y recurre al silbido que atraviesa todas las culturas (y varias especies), como código de comunicación. De este modo, el participante se enfrenta a un sistema que tiene la capacidad de dialogar con él. El participante es invitado a silbar, y luego, ese silbido es reproducido por la máquina en un acto de mímica sonora. En este caso, podemos observar que el cuerpo interactuante es el aparato fonador (y los pulmones que proveen el aire para que el silbido se genere). En relación a los umbrales perceptivos desarrollados en puntos anteriores, este trabajo sólo es sensible al silbido, no así a la palabra. Es decir, si un usuario articula el lenguaje verbal, la obra permanece inmutable; se trataría de una acción que queda fuera de los rangos de percepción de la obra.

\section{Э. Órganos}

\section{The body and the outside universe have exchanged places; the new adventure is not to discover new lands or planets but to see and map the inside of the body.}

Lev Manovich

Un tercer recorte estaría dado por el cuerpo y su integración desde los órganos. Esta categoría supone viajar más allá de la dermis e ingresar al núcleo interno del cuerpo. Los cuerpos se vuelven reversibles, plegados para invertir la relación y colocar lo más interno en sus bordes, en contacto con el mundo exterior.

\section{A) Corazón}

Podemos encontrar un conjunto de obras que trabajan con el pulso cardíaco como acción interactiva, con una particularidad: usualmente, se trata de una acción involuntaria. Rafael Lozano Hemmer explora los alcances de esta aproximación en su serie de trabajos denominados «Pulse Room» (2006), "Pulse Park» (2008), "Pulse Spiral» (2008), «Pulse Tank» (2008); en las distintas versiones hay una operación en común: el pulso cardíaco del usuario presente es traducido a impulsos lumínicos o a movimientos de agua, en espacios cerrados o inclusive abiertos. 


\section{B) Pulmones}

Obras que emplean la respiración o el soplido como acción interactiva, se conectan a la actividad pulmonar como punto de contacto con la obra. Scott Snibbe desarrolla su obra «Blow Up» (2005) donde el soplido vertebra la experiencia. Una interface conformada por una matriz de pequeños ventiladores (coolers), aguarda a que el usuario la accione. A través del soplido, el usuario hará mover las paletas de los micro-ventiladores. Ese patrón de aire será memorizado y luego replicado en una estructura análoga, de mayores dimensiones, con grandes ventiladores. El pequeño gesto se reproducirá en fuertes corrientes de aire en el lugar. Otras personas presentes en la sala podrán ubicarse delante de la matriz mayor de ventiladores y sentir en su piel, el viento diseñado por el usuario en la interface análoga. En este caso se trata de una obra que combina modos parciales de interacción/recepción.

\section{C) Cerebro}

En algunos trabajos, el fenómeno mensurable en el devenir de la forma tangible de la obra es la actividad cerebral. En «Eunoia» (2013) de Lisa Park, las ondas cerebrales son leídas para manipular el movimiento del agua en unos contenedores que rodean al cuerpo de la performer, quien interactúa en absoluta quietud externa. Las superficies vibran y el agua se despliega en patrones diferentes comandados por la intangibilidad de un proceso interno.

La obra "Neuroknitting» (2013) de Varvara Guljajeva, Mar Canet, y Sebastián Mealla, provee al usuario una interface para medir su actividad cerebral. La representación visual de las ondas conformará el patrón de tejido de una máquina que reproducirá el diseño. Concluida la experiencia, el usuario tendrá una trama física reflejo de su actividad interna, mental.

En estos casos se produce una paradoja: son cuerpos que interactúan desde un reposo aparente. Vistos desde el exterior, no distan demasiado de los cuerpos espectatoriales. Inclusive, si pensamos que sus ojos permanecen cerrados, parecen estar más próximos al sueño que a la actividad de la vigilia. Sin embargo, un proceso interactivo discurre, conectando el interior con el exterior.

\section{Conclusión}

La ingeniería de los cuerpos ha llegado al Arte, no siempre en el estado de reconfiguración física expresado claramente en las prótesis y los injertos de cuerpos extrańos; aparece una forma más sutil pero igualmente determinante para la experiencia artística: el diseño del cuerpo en la obra, la ideación de un cuerpo que será percibido por otro cuerpo (el de la máquina) y que podrá actuar bajo ciertas condiciones y en determinados contextos. Los cuerpos representados se superponen o corren en paralelo, se elevan desde el plano físico al plano simbólico.

Detrás de la ilusión óptica de cuerpos enteros en interacción, podemos ver la presencia de un cuerpo simbólico, diferente al físico en el espacio de la obra, que porta metáforas al tiempo que sensaciones. El cuerpo es contenido, en un doble sentido: contenido por los límites del diseńo; y contenido como parte inherente a la propuesta expresiva del hecho artístico en el que se inscribe. Se suele pensar que el contenido es algo que está más allá de la interface, que la interface supone la frontera entre el sujeto y los contenidos. Los cambios a los que asistimos tal vez estén señalando que ya es hora de repensar estas nociones, y advertir que el cuerpo, en el Arte Interactivo, se adiciona como recurso expresivo a la paleta del artista con nuevos medios.

\section{Referencias}

Ascott, Roy. (2010). La Trayectoria del Arte. Medios-húmedos y las Tecnologías de la Conciencia. El medio es el diseño audiovisual. Colombia: Universidad de Caldas.

Huhtamo, Erkki. (2007). Twin-Touch-Test-Redux: Media Archaeological Approach to Art, Interactivity, and Tactitility. En: Media Art Histories. London: MIT Press.

Jones, Caroline. (2005). The Mediated Sensorium. En: Sensorium. Embodied experience, technology, and contemporary art. London: MIT Press.

Machado, Arlindo. (2009). El sujeto en la pantalla. La aventura del espectador, del deseo a la acción. Barcelona: Editorial Gedisa.

Machado, Arlindo. (2000). Cuerpos y Mentes en Expansión. En: El Paisaje Mediático. Sobre el desafío de las poéticas tecnológicas. Buenos Aires: Libros del Rojas. UBA.

Manovich, Lev. (2006).Visual technologies as cognitive protheses: a short history of the externalization of the mind. En: The Prosthetic Impulse. From a Posthuman present to a biocultural future. London: MIT Press.

Pau, Alsina. (2007). Humanismo 2.0: Arte, ciencia, tecnología y sociedad. Barcelona: Editorial UOC.

Rokeby, David. The Armonics of Interaction. En línea: http://www. davidrokeby.com/harm.html

Sibilia, Paula. (2005). El hombre post-orgánico. Cuerpo, subjetividad y tecnologías digitales. México: Fondo de Cultura Económica. 